\title{
Image collection strategies for single particle cryoEM
}

Farzad Jalali-Yazdi and Eric Gouaux

OHSU, United States

Advancements in direct electron detectors, such as larger sensors and higher refresh rates, as well as data processing software, have contributed to the continuous increase in the number and resolution of cryoEM structures [1]. Underpinning the explosion in new structures are image collection strategies whereby electron beam tilt is implemented to acquire images from holes in a $\sim 5 \mu \mathrm{m}$ radius from the center of the cluster without moving the stage. Understanding of the effects of beam-tilt on image quality [2], implementation of beam tilt compensation in RELION [3] and calibration of comma versus image shift implemented in SerialEM [4], have together led to a significant increase in image acquisition rates in the past few years. Here, we discuss some parameters that users can consider when deciding on the optimal data collection strategies and magnifications. While the concepts used in this work can be generalized to other detectors and electron microscopes, the estimates presented here are for the $300 \mathrm{kV}$ Titan Krios microscopes coupled with Gatan K3 direct electron detectors.

While each microscope likely has some variation on the exact image acquisition protocol, most protocols involve the following steps:

1: Moving the stage to the center of the cluster, and waiting until stage drift has settled.

2: Estimating the defocus at that area and adjusting to the targeted defocus.

3: Tilting the beam to the collection point and waiting for the beam to settle.

4: Exposing the spot, recording typically between 40-100 frames, and saving the data.

5: Repeating steps 3-5 until all the points in the cluster have been acquired.

6: Moving to the center of the next cluster of spots.

Often the exposure time is not the most time consuming step in this process and decisions need to be made with regards to the number of images, magnification, and imaged area. The first parameter that we looked at is the number of images we can acquire per hole (Figure 1). While most often it is desirable to maximize the number of images per hole, as this increases the collection speed and reduces the total number of holes needed for a dataset, there can be circumstances where only a single image per hole is taken, such as a situation in which the ideal ice thickness can only found in the center of hole. The number of possible images per hole depends on three parameters. The magnification, the diameter of the hole, and the diameter of the beam. The beam needs to be of a certain minimum size, larger than the diagonal of the imaged area, as having the edges of the image be at the edge of the beam results in severe beam fringes being present in the acquired images. Experimentally we noticed that a beam diameter of $\sim 2.1 \mathrm{X}$ larger than the width of the image minimizes fringing at the edges. Using this ratio, we show in Figure 1 the maximum number of images that can be acquired per hole, depending on the diameter of the hole and the magnification. Note that the diameter of the beam might have to be increased in the case that significant beam fringes are present, resulting in potentially lower number of acquisitions per hole.

Our collections typically involve $\sim 50$ frames per acquisition, without gain-correction, recorded in TIFF format and at super-resolution. We recorded the amount of time each step typically took, and were able to 
estimate the number of images we could acquire per hour. In our approach, it generally took $\sim 60$ seconds for stage movement, drift settlement, defocus estimation and calibration. The exposure time depends on total exposure target (here set to $50 \mathrm{e} / \AA 22$ ) and the magnification. To minimize coincident loss [5,6], the exposure was limited to $20 \mathrm{e} / \mathrm{pix} / \mathrm{s}$ on the $\mathrm{K} 3 \mathrm{We}$ also noticed that it took an average of 4 seconds for the image to be saved on our drives. These estimates combined with the number of images per hole resulted in the calculated image acquisition rates shown in Figure 2.

Direct electron detectors' DQE is lower when the resolution approaches the Nyquist frequency. For samples in optimal ice thickness or with a high degree of symmetry, it is possible exceed the Nyquist frequency during data processing [5], however thus far for most membrane proteins, this feat has not been achieved. Therefore the choice for the correct magnification to use depends on several factors such as the size of the sample being imaged and the estimated resolution that the sample will achieve.

As a starting approximation, if we assume that sample resolution will not be limited by DQE, maximizing the number of particles acquired per unit time leads to optimal microscope use. In our calculations we show that for the $1.2 / 1.3 \mu \mathrm{m}$ holy carbon grids, there is a proportional relation between imaged area per hour and magnification, where with increasing magnification there is a decrease in the number of particles obtained per unit time. Interestingly, when using the $2 \mu \mathrm{m}$ diameter holes, there is virtually no difference between the area imaged per unit time when using the 1.65 or $1.39 \AA$ /pix scales. We noticed that this transition, where going to a higher magnification does not impact imaged area per unit time, generally happens when transitioning from a single image to multiple images per hole for the first time. In these instances, barring any computational resource limitations, it is advantageous to use the higher magnification as it does not lead to a lower particle acquisition per unit time, the micrographs will have a higher DQE, and the total number of holes required for acquisition will drop, reducing the number of squares or grids needed for a collection.

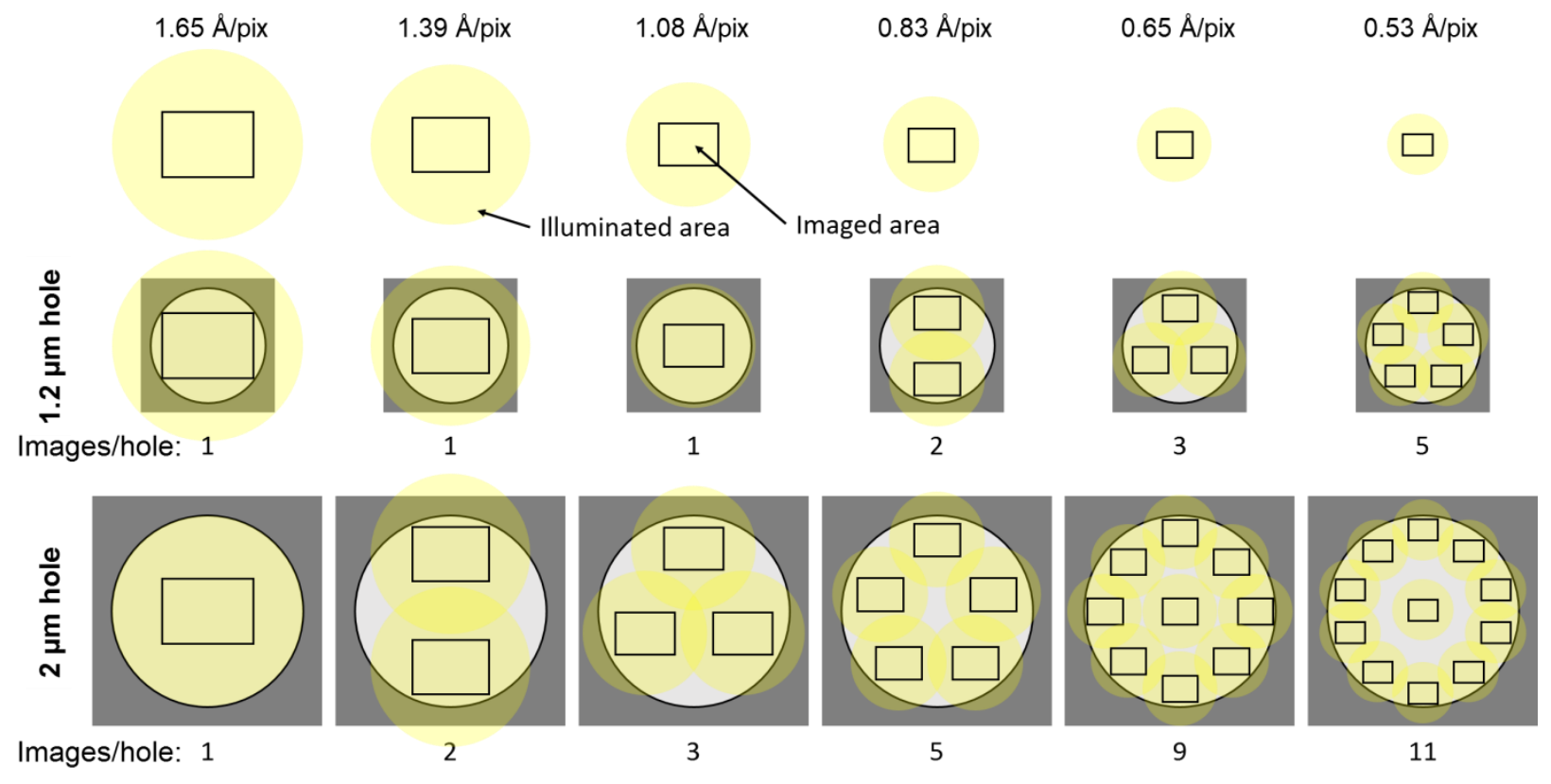

Figure 1. Configuration of multiple images per hole based on hole diameter, illuminated area, and the K3 sensor imaged area 


\begin{tabular}{|c|c|c|c|c|c|c|c|c|c|c|c|c|c|c|c|c|c|c|}
\hline \multirow[b]{2}{*}{ Physical pixel scale (A/Pix) } & \multicolumn{6}{|c|}{$1.2 / 1.3 \mu \mathrm{m}$ grids } & \multicolumn{6}{|c|}{$2 / 2 \mu \mathrm{m}$ grids } & \multicolumn{6}{|c|}{$2 / 1 \mu \mathrm{m}$ grids } \\
\hline & 1.65 & 1.39 & 1.08 & 0.83 & 0.65 & 0.53 & 1.65 & 1.39 & 1.08 & 0.83 & 0.65 & 0.53 & 1.65 & 1.39 & 1.08 & 0.83 & 0.65 & 0.53 \\
\hline Beam diameter $(\mu \mathrm{m})$ & 2.0 & 1.7 & 1.3 & 1.0 & 0.78 & 0.64 & 2.0 & 1.7 & 1.3 & 1.0 & 0.8 & 0.6 & 2.0 & 1.7 & 1.3 & 1.0 & 0.8 & 0.6 \\
\hline \# of images / hole & 1 & 1 & 1 & 2 & 3 & 5 & 1 & 2 & 3 & 5 & 9 & 11 & 1 & 2 & 3 & 5 & 9 & 11 \\
\hline \# of holes / stage move & 16 & 16 & 16 & 16 & 16 & 16 & 5 & 5 & 5 & 5 & 5 & 5 & 9 & 9 & 9 & 9 & 9 & 9 \\
\hline \# of images per stage move & 16 & 16 & 16 & 32 & 48 & 80 & 5 & 10 & 15 & 25 & 45 & 55 & 9 & 18 & 27 & 45 & 81 & 99 \\
\hline Dose rate $\left(e / \AA^{2} / s\right)$ & 12 & 14 & 19 & 24 & 31 & 38 & 12 & 14 & 19 & 24 & 31 & 38 & 12 & 14 & 19 & 24 & 31 & 38 \\
\hline Exposure time (s) & 4.1 & 3.5 & 2.7 & 2.1 & 1.6 & 1.3 & 4.1 & 3.5 & 2.7 & 2.1 & 1.6 & 1.3 & 4.1 & 3.5 & 2.7 & 2.1 & 1.6 & 1.3 \\
\hline Image acquisition Rate per hour & 303 & 321 & 344 & 453 & 524 & 593 & 179 & 267 & 336 & 425 & 517 & 561 & 243 & 333 & 403 & 486 & 566 & 607 \\
\hline Imaged area $\left(\mu \mathrm{m}^{2}\right)$ per hour & 195 & 146 & 95 & 74 & 52 & 39 & 115 & 122 & 92 & 69 & 52 & 37 & 156 & 152 & 111 & 79 & 56 & 40 \\
\hline Holes imaged per hour & 303 & 321 & 344 & 266 & 175 & 119 & 179 & 134 & 112 & 85 & 57 & 51 & 243 & 167 & 134 & 97 & 63 & 55 \\
\hline Reduction in acquisition rate in CDS mode & $26 \%$ & $24 \%$ & $21 \%$ & $21 \%$ & $19 \%$ & $18 \%$ & $17 \%$ & $21 \%$ & $20 \%$ & $20 \%$ & $19 \%$ & $17 \%$ & $22 \%$ & $24 \%$ & $23 \%$ & $22 \%$ & $20 \%$ & $18 \%$ \\
\hline
\end{tabular}

Figure 2. Maximum collection rate depending on hole diameter and magnification

\section{References}

[1] E Callaway, Nature 578 (2020), p. 201.

[2] A Cheng et al., Journal of structural biology 204 (2018), p. 270.

[3] J Zivanov et al., eLife 7 (2018), p.

[4] DN Mastronarde, Journal of structural biology 152 (2005), p. 36.

[5] M Sun et al., bioRxiv (2020), p.

[6] JR Feathers et al., bioRxiv (2019), p. 